\title{
Three-axis Fiber-optic Body Force Sensor for Flexible Manipulators
}

\author{
Yohan Noh, Sina Sareh, Helge Würdemann, Hongbin Liu, James Housden, \\ Kawal Rhode, Kaspar Althoefer
}

\begin{abstract}
This paper proposes a force/torque sensor structure that can be easily integrated with a flexible manipulator structure. The sensor's ring-like structure with its hollow inner section provides ample space for auxiliary components, such as cables and tubes, to be passed through and, hence, is very suitable for integration with tendon-driven and fluid-actuated manipulators. The sensor structure can also accommodate the wiring for a distributed sensor system as well as for diagnostic instruments that may be incorporated in the manipulator. Employing a sensing approach based on optical fibers as done here allows for the creation of sensors that are free of electrical currents at the point of sensing and immune to magnetic fields. These sensors are inherently safe when used in the close vicinity of humans and their measuring performance is not impaired when they are operated in or nearby machines such as magnetic resonance imaging (MRI) scanners. This type of sensor concept is particularly suitable for inclusion in instruments and robotic tools for minimally invasive surgery (MIS). The paper summarizes the design, integration challenges and calibration of the proposed optical three-axis force sensor. The experimental results confirm the effectiveness of our optical sensing approach and show that after calibrating its stiffness matrix, force and momentum components can be determined accurately.
\end{abstract}

\section{INTRODUCTION}

Force/torque sensor development and integration is an important element in the process of creating flexible robot structures. Such sensors provide a useful means of measuring the forces and moments imparted on the robot structure especially important where robot arms operate in the close vicinity of humans, as is the case in robot-assisted minimally invasive surgery (RMIS). Equipping a robot arm with force/torque sensors ensures the arm's efficient steering and control and prevents the application of excessive forces in the restrictive surgical environment. In order to overcome the shortcomings of conventional medical devices and recently emerging surgical robots, in particular those that are composed of rigid links, researchers have started developing flexible manipulators. Taylor and Choset developed

Manuscript received Jan 31st, 2015. The research leading to these results has received funding from the European Commission's Seventh Framework Programme; project STIFF-FLOP (Grant No: 287728).

Yohan Noh, Sina Sareh, Würdemann Helge, Hongbin Liu, and Kaspar Althoefer are with King's College London, School of Natural and Mathematical Sciences, Department of Informatics, UK

(e-mail corresponding author: yohan.noh@kcl.ac.jp)

James Housden, and Kawal Rhode are with the Department of Biomedical Engineering, Division of Imaging Sciences and Biomedical Engineering, King's College London, UK dexterous, flexible snake-like manipulators that can provide relatively high dexterity or mobility in confined spaces that may not be easily reached by the traditional class of robotic instruments (Amir Degani et al., 2006; Takeyoshi Ota al., 2008; Simaan et al., 2009; Alexander et al., 2008) [1-4]. Webster III et al., developed active cannula manipulators which are a new class of thin, dexterous continuum robots, capable of accessing narrow openings such as the throat and lung (D. C. Rucker et al., 2010; Zhang et al., 2009) [5-8]. Yang et al, developed an articulated universal joint-based flexible manipulator by locating the actuators at the distal tip and embedding a micro motor. This system improves access and distal dexterity, it can operate along curved instrument pathways and enhances manipulation accuracy and stability (Shang et al., 2010) [9-11]. Dankelman et al. developed flexible manipulators which can control rigidity or an aiding shaft with controllable rigidity in or around the medical device. The shaft can be changed from rigid to compliant as appropriate for each phase of the intervention (Arjo et al., 2010) [12]. Setapen developed a flexible manipulator which can change its stiffness and is capable of dexterous precise motion control using cables (Cheng et al. 2012) [13]. However, most of these recently developed surgical robotic devices do not incorporate sensors capable of providing information about the devices' physical interactions with the environment.

In this paper, drawing from our expertise in optic fiber sensing [14-18] we present an integrated sensing solution

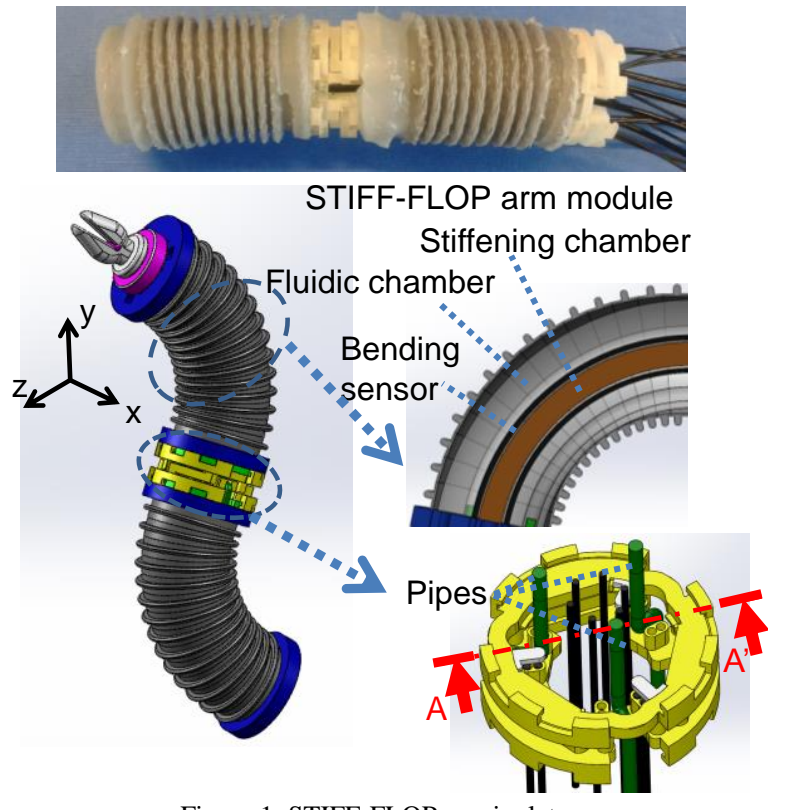

Figure 1. STIFF-FLOP manipulator 


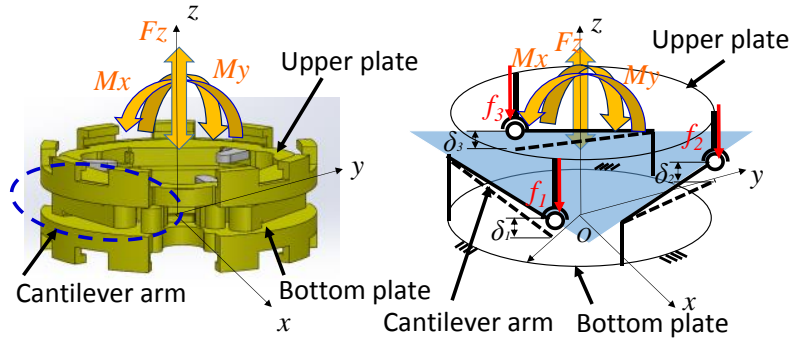

Figure 2. Measurable force and moment components on the three-axis force sensor and simplified

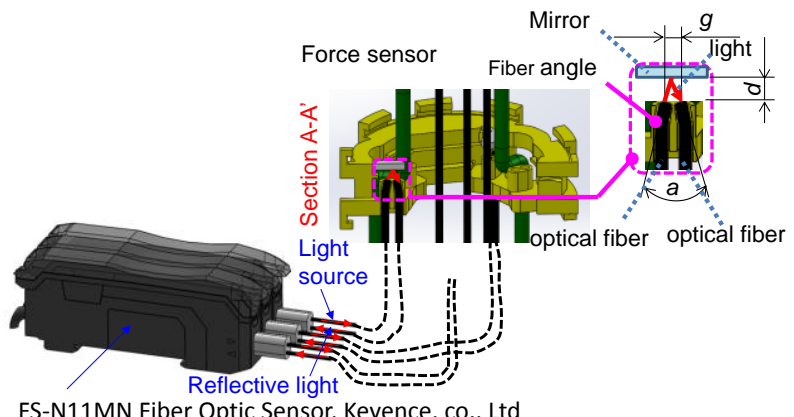

FS-N11MN Fiber Optic Sensor, Keyence, co., Ltd

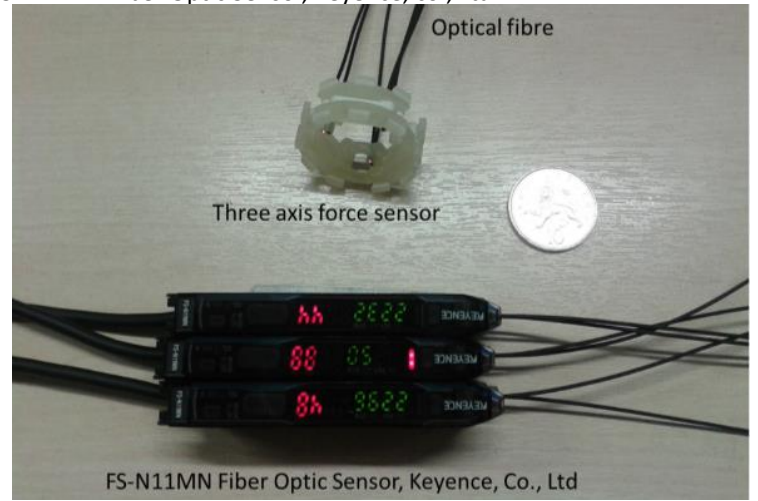

Figure 3. Three pairs of the optical fibers with The FS-N11MN Fiber Optic Sensor

TABLE I. MEASURABLE RANGE OF FORCE $F_{Z}$, AND MOMENTS $M_{X}$ AND $M_{Y}$

\begin{tabular}{|c|c|}
\hline Force and Moment ranges & $\begin{array}{c}\text { Sensor structure size, } \\
\text { height } H \text { and diameter } D\end{array}$ \\
\hline$F_{z}+/-3 \mathrm{~N}$ & $H=14 \mathrm{~mm}$ \\
\hline$M_{x}+/-3.0 \mathrm{Ncm}$ & $D=30 \mathrm{~mm}$ \\
\hline$M_{y}+/-3.0 \mathrm{Ncm}$ & \\
\hline
\end{tabular}

which allows flexible robot arms to perceive their physical interactions with the environment by measuring force and torque applied to the robot's body.

In contrast to other sensing methods, optic fiber based sensing has a number of advantages: it is safe (no electrical currents at the point of measurement); can be miniaturized; it is extendable, that is light information can be transmitted over large distances; it is low-cost, and last but not least it is magnetic resonance compatible which means that it can be used during minimally invasive surgery performed in a magnetic resonance scanner $[16,19]$. One example of an MRI compatible force/torque sensor was developed by Monfaredi et al. [20] using FBG (Fiber-Bragg-Grating) sensors. This has two degrees of freedom and is designed for use in MRI-guided prostate interventions.

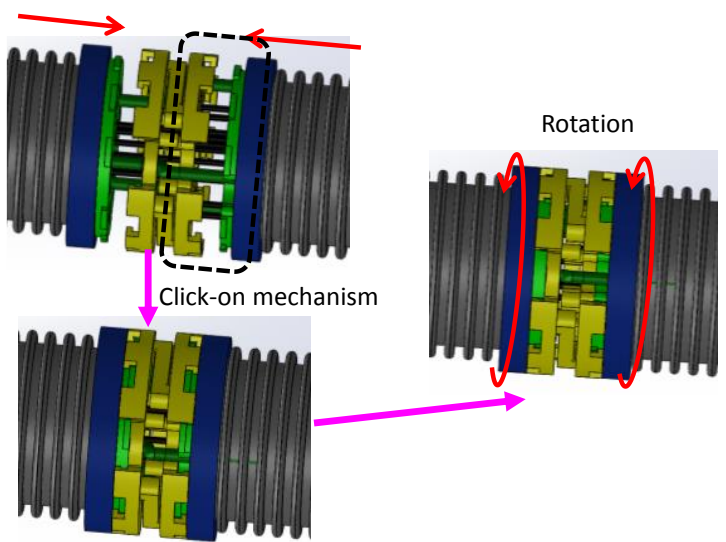

Figure 4. The STIFF-FLOP manipulator and click-on mechanism

In this paper, we instead exploit the principle of light intensity modulation (LIM) in optic fibers. A ring-shaped multi-axis force sensor (as shown in Figures 1 to 3 ) is designed and implemented allowing to measure force $F_{z}$ (force applied perpendicular to the sensor structure), torque $M_{y}$ (torque around the $\mathrm{y}$ axis) and torque $M_{x}$ (torque around the $\mathrm{x}$ axis) (Figure 2). The sensor's structure is designed to fit in the structure of cylindrically-shaped robot arms. It is noted that the sensor concept is generic, can be employed in a range of cylindrical robot arms and it is capable of measuring forces and moments transmitted between adjacent links.

We explain the design and calibration of this multi-DOF force/torque sensor with its ring-like structure and its hollow inner section which provides ample space for auxiliary components. Our method will be applied to the STIFF-FLOP arm which represents a prime example of a flexible manipulator [21-24]. In addition, we obtain the sensor's stiffness matrix from our experimental study, and validate the obtained stiffness matrix. Our study also evaluates various properties such as error measurement, repeatability, hysteresis, and crosstalk.

\section{DESIGN MethodS AND FABRICATION}

\section{A. Design Considerations}

To be useful in robotic surgery, the multi-axial force sensor should satisfy several conditions:

1) It should be capable of measuring forces and moments in a range suitable for the application: based on the following papers [25-27], the measurable range of the force applied by robotic surgical systems ranges from 0 to $21 \mathrm{~N}$.

2) It should be miniaturisable: sensor structure (diameter) should be less than $15 \mathrm{~mm}$ for current commercialized trocars.

3) It should be easily adaptable and able to be conceptually integrated with a wide range of robot arms

4) It should affect the robot structure as little as possible and provide space for necessary tubes and cables for other functions, including actuation, sensing, attached tool actuation, and

5) It should be MRI compatible.

In this paper, to prove the efficiency of our proposed 
three-axis force sensor for flexible manipulators, we designed the first prototype with a measurement range and size as shown in Table 1.

\section{B. Configuration of Optical Multi Axis Force Sensor}

The structure of the proposed three-axis force sensor is shown in Figures 1 to 3. The sensor uses quarter-millimeter diameter optical fibers (SH1001-1.0 from LasIRvis Co. Ltd, wave length: red 4-element LED (wavelength $630 \mathrm{~nm}$ ), outer diameter of jacket: $1.0 \mathrm{~mm}$ ); mirrors are incorporated in the structure to act as reflective surfaces; a flexible ring-like structure made of ABS plastic using rapid prototyping is created deforming in response to force component $F_{z}$ and the two moment components, $M_{x}$ and $M_{y}[15,23]$. In order to measure these three force and moment components, at least three flexures (cantilever arms) are required, as illustrated in Figure 2. In order to measure the three components of the force and the moments, the three deflections $\delta_{1}, \delta_{2}$, and $\delta_{3}$ of the sensor's structure are measured by way of three pairs of optical fibers. The components, are symmetrically located at $120^{\circ}$ at the same radial distance (Figure 1 and 5). The three pairs of optical fibers are fixed into the three elliptical parts of the sensor structure, as shown in Figure 5 (transparent blue color of upper image). The three pairs of optical fibers can also be fixed alternately into the other three white elliptical parts.

In each fiber pair, one fiber emits light, while the other one receives light via the reflective surface on the corresponding fixture: the light intensity of the reflected light is modulated by the applied forces and moments displacing the fixture and, in turn, the reflective surface (Figures 1 and 3). Fibers are arranged as follows: the tips of the transmitting and receiving fibers are positioned inside the sensor structure in a way that optimizes the light transmission via the reflective surface. The measured light intensity is a function of three parameters, distance $d$, angle $a$, and gap $g$ (Figure 3 ).

The receiving fibers' proximal ends are connected to KEYENCETM Digital Fiber Sensors FS-N11MN converting the amount of reflective light into voltage (Figure 3). When external forces and moments are applied to the upper plate, the three associated cantilever arms will be deflected (Figure 2 ). The three corresponding receiving fibers allow measuring the resultant cantilever arm deflections $\left(\delta_{1}, \delta_{2}\right.$, and $\left.\delta_{3}\right)$ between the upper plate and the bottom plate (Figure 2). The deflections $\delta_{1}, \delta_{2}$, and $\delta_{3}$ can be determined from the output voltages that represent the light intensity emitted from the receiving fibers.

In order to anchor the force sensor to the structure of the associated manipulator arm, a click-on mechanism is adopted, as shown in Figures 1 and 4. The ring-like hollow sensor structure allows the passing-through of optical fibers as well as of other wirings and tubings between the different segments of the robot.

\section{Optical Fiber Optimization, Modeling and Simulation of Sensor Structure}

The structure of our ring-shaped multi-axis force sensor

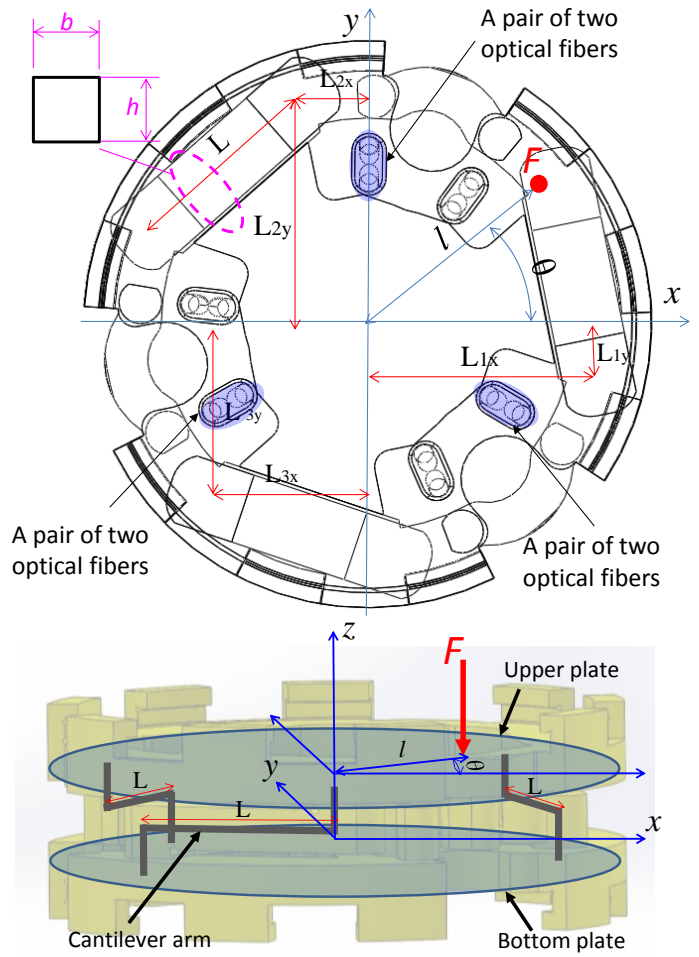

Figure 5. Sensor structure (section of cantilever beam) dimension and design variables

with its three cantilever arms is shown in Figures 2 (right) and 5. When a load is applied to the upper plate of the sensor structure three apparent force components $\left(F_{z}, M_{x}\right.$ and $\left.M_{y}\right)$ are experienced at the three cantilevers in the form of force components $f_{1}, f_{2}$, and $f_{3}$ (Figure 2). The three transmitted forces cause each of the three cantilever beams to deflect ( $\delta_{l}$, $\delta_{2}$, and $\delta_{3}$ ). The transmitted forces $f_{1}, f_{2}$, and $f_{3}$ can be calculated by multiplying spring coefficient $\left(k_{1}, k_{2}\right.$, and $\left.k_{3}\right)$ and the deflections $\left(\delta_{1}, \delta_{2}\right.$, and $\left.\delta_{3}\right)$ of the three cantilever arms:

$$
\begin{gathered}
f_{1}=k_{1} \delta_{1} \\
f_{2}=k_{2} \delta_{2} \\
f_{3}=k_{3} \delta_{3}
\end{gathered}
$$

Employing standard models describing the behavior of material under strain [28], the deflection of the cantilever arm is calculated as follows (Figure 5):

$$
\begin{gathered}
I=\frac{b h^{3}}{12} \\
\delta=\frac{f L^{3}}{3 E I} \\
k=\frac{3 E I}{L^{3}}
\end{gathered}
$$

where spring coefficient $k$ is a function of the distance away from the hinge, $L$, the modulus of elasticity, $E$, the moment of inertia, $I$, the width of arm' section, $b$, the section of arm' section, $h$. 


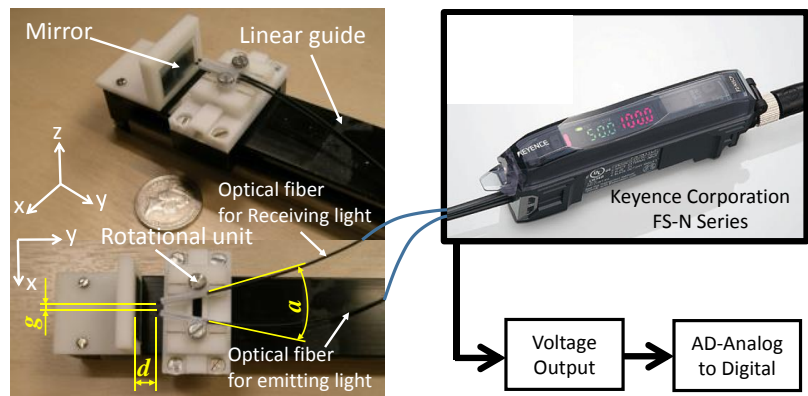

Figure 6. Experiment setup to investigate the effects of the angle of the optical fiber pair and the distance from the fiber tip to the mirror

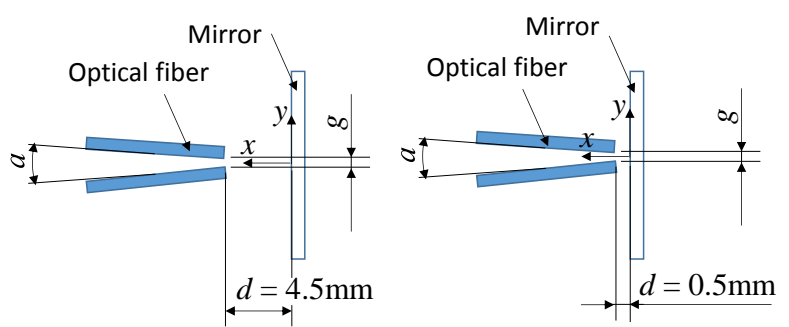

Figure 7. Experiment setup image of the optical fibers as shown in Fig. 6

When an amount of external force and moments are applied to the upper plate as shown in Figures 2 and 5, force $F_{z}$ and moment components $M_{x}$ and $M_{y}$ can be calculated by substituting force components $f_{1}, f_{2}$, and $f_{3}$ into the following set of equations:

$$
\begin{aligned}
& F_{z}=f_{1}+f_{2}+f_{3} \\
& M_{x}=F \cdot l \cdot \sin \theta=L_{1 y} \cdot f_{1}-L_{2 y} \cdot f_{2}+L_{3 y} \cdot f_{3} \\
& M_{y}=F \cdot l \cdot \cos \theta=L_{1 x} \cdot f_{1}-L_{2 x} \cdot f_{2}-L_{3 x} \cdot f_{3}
\end{aligned}
$$

The deflections of the three cantilever arms are represented in turn by the output voltages $\left(v_{1}, v_{2}\right.$, and $\left.v_{3}\right)$ of the three fiber pairs, and the forces are computed as follows:

$$
\begin{aligned}
& f_{1}=k_{1} \delta_{1}=m_{1} v_{1} \\
& f_{2}=k_{2} \delta_{2}=m_{2} v_{2} \\
& f_{3}=k_{3} \delta_{3}=m_{3} v_{3}
\end{aligned}
$$

where, $m_{1}, m_{2}$, and $m_{3}$ are obtained by means of calibration. Finally, from Eqs. 10-12, external force $F_{z}$ and two moment components $M_{x}$ and $M_{y}$ can be calculated from $f_{1}, f_{2}$ and $f_{3}$, and stiffness matrix $\boldsymbol{k}$ can be obtained as follows:

$$
\left[\begin{array}{c}
F_{z} \\
M_{x} \\
M_{y}
\end{array}\right]=\boldsymbol{k} \cdot \boldsymbol{v}=\left[\begin{array}{ccc}
m_{1} & m_{2} & m_{3} \\
m_{1} L_{1 y} & -m_{2} L_{2 y} & m_{3} L_{3 y} \\
m_{1} L_{1 x} & -m_{2} L_{2 x} & -m_{3} L_{3 x}
\end{array}\right] \cdot\left[\begin{array}{c}
v_{1} \\
v_{2} \\
v_{3}
\end{array}\right]
$$

1) Optimization of Characteristic curves on a pair of optical fibers

In order to develop an optical based force/torque sensor, the following key points should be considered: (1) relatively

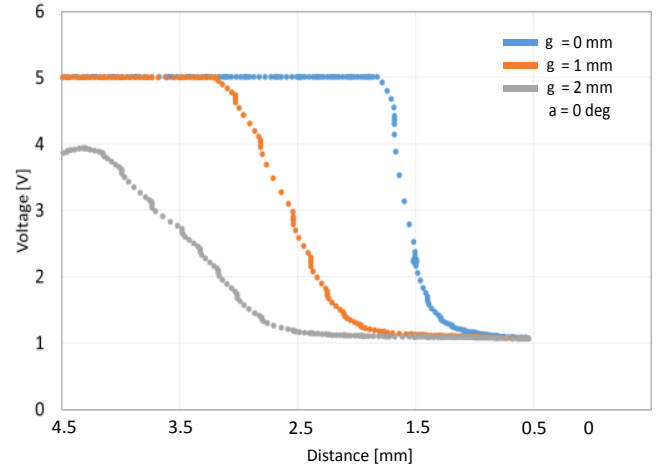

Figure 8. Characteristic curve of output voltage of optical fiber in case of $a=0^{\circ}$ and changing $g$

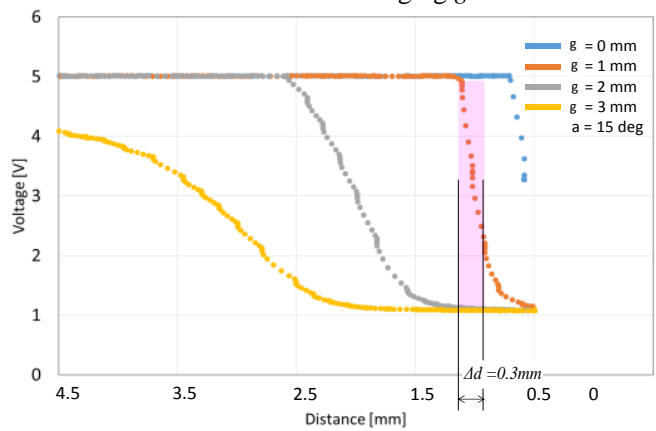

Figure 9. Characteristic curve of output voltage of optical fiber in case of $a=15^{\circ}$ and changing $g$

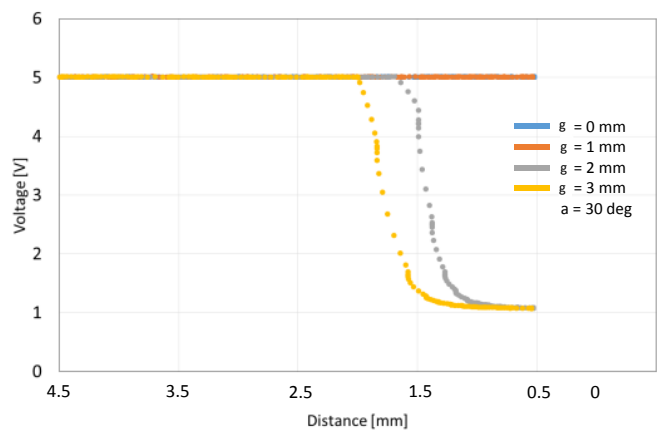

Figure 10. Characteristic curve of output voltage of optical fiber in case of $a=30^{\circ}$ and changing $g$

small deformations resulting from externally applied forces and moments should produce large voltage changes at the KEYENCETM Digital Fiber Sensor to achieve a large measurement range, and (2) the measured voltage values should ideally vary linearly as a function of the distance between the optical fiber tips and the corresponding reflective surface (mirror) across the measurement range.

Depending on (i) the distance $d$ between the mirror and the pair of fibers, (ii) the orientation angle $a$ between the two optical fibers, and (iii) the gap $g$ between the fibers (Figure 3 and 6), the characteristic curve between the output voltage of the optical fiber and distance $d$ was found to be as shown in Figures 8 to 10 . In order to satisfy the two key points for the development of the three-axis force sensor (as outlined above), a set of experiments were carried out to find the best angle $a$, gab $g$, distance $d$ using a testing device. This fiber adjustment device consists of a linear guide, a rotational unit, a mirror, a linear guide, and an actuator, as shown in Figures 6 


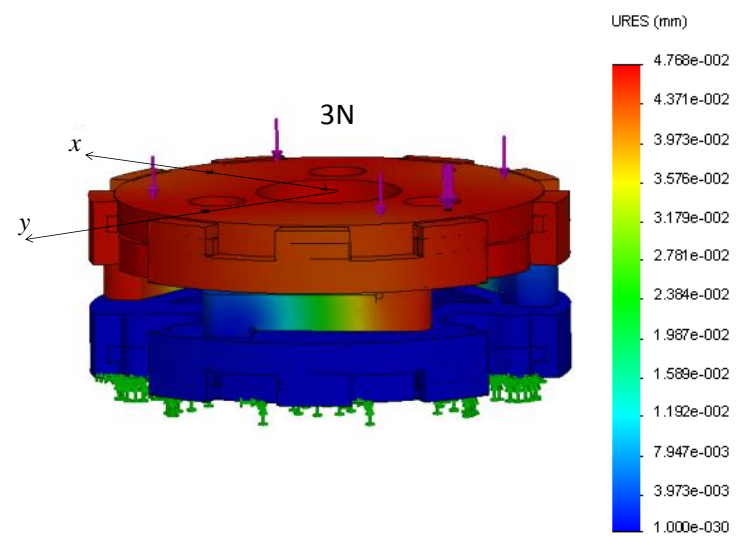

Figure 11. FEM Simulation performed with the Solidworks Simulation tool $F_{z}=-3 \mathrm{~N}$

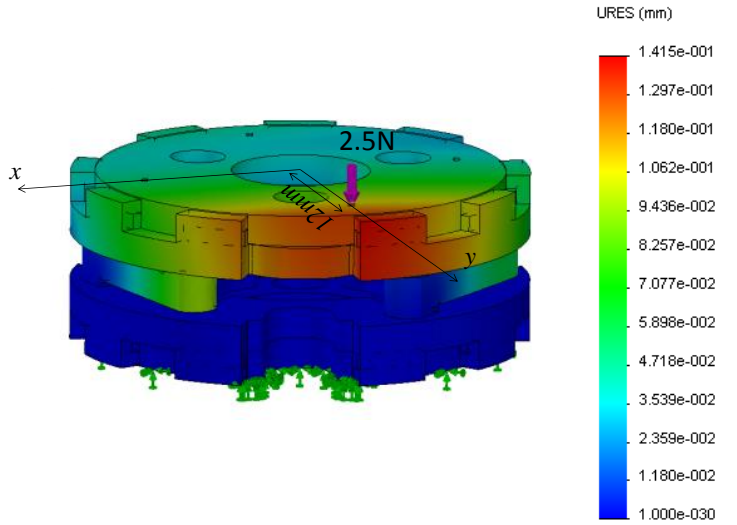

Figure 12. FEM Simulation performed with the Solidworks Simulation tool $F_{z}=-2.5 \mathrm{~N}$ and $M_{x}=-3.0 \mathrm{Ncm}$

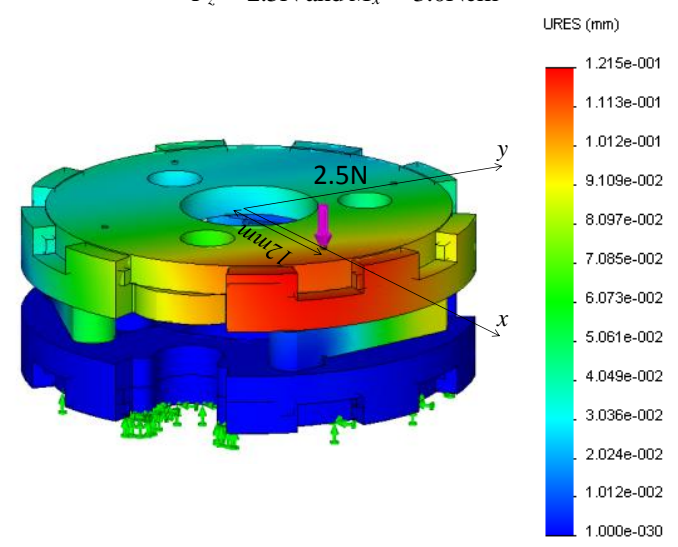

Figure 13. FEM Simulation performed with the Solidworks Simulation $F_{z}=-2.5 \mathrm{~N}$ and $M_{y}=3.0 \mathrm{Ncm}$

and 7. The device allows varying angle $a$, gap $g$, and distance $d$ (Figure 7). Whilst varying the three parameters, the output voltages of the Keyence sensors are recorded by Analog-Digital Convertors (ADCs). By conducting a set of experiments and adjusting the parameters over their respective ranges, $a \in\left\{0^{\circ}, 15^{\circ}, 30^{\circ}\right\}, g \in\{0,1,2,3 \mathrm{~mm}\}$ and $0.5 \mathrm{~mm} \leq d \leq 4.5 \mathrm{~mm}$, the characteristic curves for the fiber-based sensors can be determined. For the first experiments $a$ was kept constant at $0^{\circ}$. Figure 8 shows profiles of output voltage versus the distance to the mirror when $a=0^{\circ}$, and at three different gaps $g$. It can be seen that in the vicinity of the mirror, the output voltage of the optical fiber suddenly changes from $1 \mathrm{~V}$ to $5 \mathrm{~V}$. For smaller values of gap $g$, the output voltage of the optical fiber demonstrated a sharp nearly linear change in the vicinity of the mirror. As shown in Figures 9 and 10, when angles $a$, and gaps $g$ are adjusted, the output voltage of the optical fiber changes from 1 to $5 \mathrm{~V}$. We observe that smaller gaps $g$ accompanied with larger values of angles $a$ result in an improved linearity in the voltage-distance profile. Note that in Figure 10, the voltage change was not observed at a distance of $0.5 \mathrm{~mm}$ with respect to the mirror, since the recorded output voltage was in the saturated region for this case. From the results of the experiments, the design of the three-axis force sensor can be attempted. In order to satisfy the two key points of the force sensor development (as outlined above in the optimization of characteristic curves on a pair of optical fibers), the characteristic curve $g=1 \mathrm{~mm}$ and $a=15^{\circ}$ (orange curve) can be used as shown in Figure 9 providing sufficient linearity and producing a $3 \mathrm{~V}$ change in response to a change of distance of $d$ of $0.3 \mathrm{~mm}$. Although the other characteristic curves also provide good opportunities for the design, they are highly non-linear, and are too steep with respect to tiny cantilever deformation, causing the output voltage to be saturated easily.

\section{2) Model and Simulation for Sensor Structure}

Depending on the measurement range of the force and

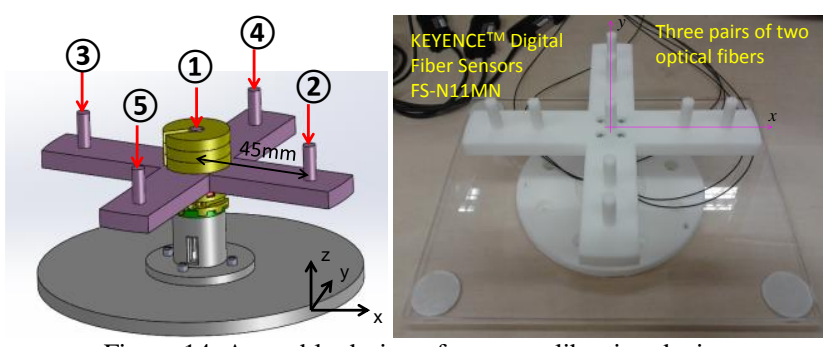

Figure 14. Assembly design of sensor calibration device

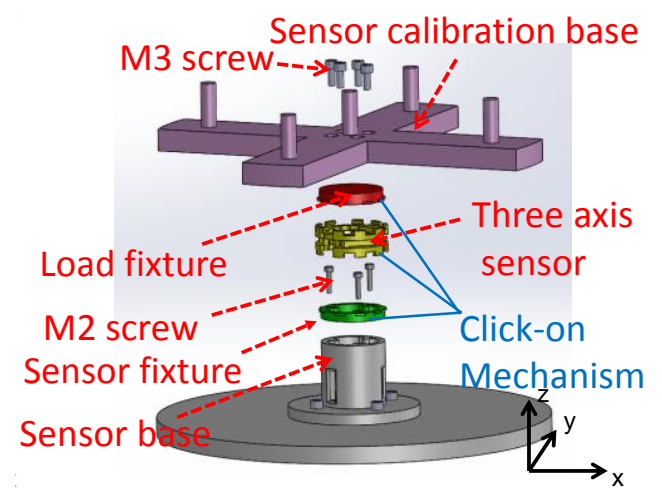

Figure 15. Assembly design of sensor calibration device showing individual components

TABLE II. EXPERIMENTAL CONFIGURATION OF THE APPLIED LOADS FOR CALIBRATION

\begin{tabular}{|c|c|c|}
\hline $\begin{array}{c}\text { Positions on calibration } \\
\text { base (see Fig. 14) }\end{array}$ & $\begin{array}{c}\text { Force and Moment } \\
\text { component }\end{array}$ & Force ranges \\
\hline 1 & $F_{z}$ & 0 to $-3 \mathrm{~N}$ \\
\hline 2 & $F_{z} M_{y}$ & -0.7 to $0 \mathrm{~N} 0$ to $3.15 \mathrm{Ncm}$ \\
\hline 3 & $F_{z} M_{y}$ & -0.7 to $0 \mathrm{~N}-3.15$ to $0 \mathrm{Ncm}$ \\
\hline (4) & $F_{z} M_{x}$ & -0.7 to $0 \mathrm{~N}-3.15$ to $0 \mathrm{Ncm}$ \\
\hline 5 & $F_{z} M_{x}$ & -0.7 to $0 \mathrm{~N} 0$ to $3.15 \mathrm{Ncm}$ \\
\hline
\end{tabular}


moments, the sensor structure can be designed by choosing design variables $L, E, I, b$, and $h$ as shown in Eqs. 4 to 6 and Figure 5.

When an amount of external force is applied to the upper plate as shown in Figure 2, each of the three cantilever arm deflections should not exceed $\Delta d=0.3 \mathrm{~mm}$ (Figure 9) because the output voltage of the pair of optical fibers has good linearity and sensitivity within this distance change. In addition, force and moment components have positive and negative values, so the default output voltage of a pair of optical fibers (no force applied) should be in the middle of the voltage range corresponding to the mid-position of $\Delta d \epsilon$ [-0.15mm to $+0.15 \mathrm{~mm}$ ] (Figure 9).

In order to determine whether the required force range for our structure can be achieved, we have performed a detailed FEA analysis. Figures 11 to 13 show the results of the FEA simulation using SolidWorks Simulation tool. The cantilever arm deflections were constrained not to exceed the range of $\Delta d$. The simulated sensor structure is then found to deform as required in response to the applied forces within the force measurement range shown in Table 1 . The values associated with material properties in this simulation were set at: tensile modulus of $1283000000 \mathrm{~N} / \mathrm{m}^{2}$, mass density of $1020 \mathrm{~kg} / \mathrm{m}^{3}$, tensile strength of $42400000 \mathrm{~N} / \mathrm{m}^{2}$; these assumptions were based on information provided by the manufacturer of the rapid prototyping machine, PROJET VisiJet ${ }^{\circledR}$ EX200, 3D SYSTEM Co., Ltd, used.

\section{SENSOR CALIBRATION}

\section{A. Setup for Calibration Experiments}

In order to use a fabricated sensor prototype as a force sensor, calibration is required. The calibration process is needed to obtain the relationship between the set of the three output voltages acquired from the three pairs of optical fibers using the KEYENCETM Digital Fiber Sensor, and the actual force and moment components $\left(F_{z}, M_{x}, M_{y}\right)$ applied to the sensor. We have designed and built a calibration device and calculated the stiffness matrix from the calibration data as described below.

\section{1) Calibration device}

The calibration device consists of a sensor calibration base, a sensor base, a load fixture and a sensor fixture. The developed three-axis force sensor was mounted on the sensor fixture, while the sensor calibration base is mounted on the load fixture, as shown in Figures 14 and 15. The sensor calibration base can exert three conditions of the force and moments for the calibration as followed: 1) only $F_{z}$ : (1), 2) $F_{z}$ and $M_{x}$ (expressed as $\left.\left(F_{z} M_{\mathrm{x}}\right)\right)$ : (4) or (5), 3) $F_{z}$ and $M_{y}$ (expressed as $\left.\left(F_{z} M_{\mathrm{y}}\right)\right)$ : (2) or (3). It is noted that cases 2) or 3) always involve a combination of force and moments. This calibration device calibrates the sensor as well as allowing the evaluation of its properties such as cross-talk, hysteresis, repeatability and error. This calibration device uses an analogue-digital convertor (ADC) and associated software to obtain the characteristic curves describing the relationship between output voltages from the optical fiber and the physical loads.

2) Calibration for $F_{z}, M_{x}$, and $M_{y}$

In the first instance, a set of loads $(0 \mathrm{~N}$ to $-3 \mathrm{~N}$ in steps of $-0.5 \mathrm{~N})$ were mounted onto the sensor calibration base; then the loads were removed again in steps of $-0.5 \mathrm{~N}$ from point (1) (see Figure 14, and Table 2). The characteristic curve showing the relationship between the loads in $\mathrm{z}$ direction $\left(F_{z}\right)$ and the output voltages from the fibers of the force sensor were recorded. This loading and unloading process was repeated three times. The characteristic curves were obtained (Figure 16). It can be seen that the characteristic curves are approximately linear.

Similar experiments were conducted to calibrate the force and moment $\left(F_{z} M_{\mathrm{x}}\right)$ and $\left(F_{z} M_{\mathrm{y}}\right)$ respectively. As part of the calibration experiments weights were added to and removed from points (4), (5)) and (2), (3) of the calibration device in increments of $(-0.1 \mathrm{~N}, \pm 0.45 \mathrm{Ncm})$ in the range of $(0 \mathrm{~N}, 0 \mathrm{Ncm})$ to $(-0.7 \mathrm{~N}, \pm 3.15 \mathrm{Ncm})$ to calculate $\left(F_{z} M_{\mathrm{x}}\right)$ and $\left(F_{z} M_{\mathrm{y}}\right)$, respectively (see Figure 14 and Table 2). Each loading/unloading cycle was repeated 3 times. The characteristic curves were plotted for all the conducted calibration experiments (see Figure $17\left(F_{z} M_{\mathrm{x}}\right)$ and Figure 18 $\left(F_{z} M_{\mathrm{y}}\right)$ respectively).

\section{B. Calculation of stiffness matrix by multiple linear regression}

All multi-axis sensors have a degree of cross coupling, a condition where the load on one axis produces a change in output on other axes. Here, each optical fiber sensor is individually calibrated with loads on each axis. The calibration data is used to generate a calibration and stiffness matrix which in turn is used to convert the output voltages to force and moment loading data. The three-by-three stiffness matrix is multiplied by the three-element voltage vector (column) to obtain the calibrated, decoupled output:

$$
\begin{gathered}
{\left[\begin{array}{c}
\text { Stiffness } \\
\text { Coefficien } t \\
\text { Matrix }
\end{array}\right] \times\left[\begin{array}{c}
\text { Sensor } \\
\text { Output } \\
\text { Volts }
\end{array}\right]=\left[\begin{array}{c}
\text { Force } \\
\text { and } \\
\text { Moments }
\end{array}\right],} \\
{\left[\begin{array}{lll}
k_{1,1} & k_{1,2} & k_{1,3} \\
k_{2,1} & k_{2,2} & k_{2,3} \\
k_{3,1} & k_{3,2} & k_{3,3}
\end{array}\right] \times\left[\begin{array}{c}
v_{1} \\
v_{2} \\
v_{3}
\end{array}\right]=\left[\begin{array}{c}
F z \\
M x \\
M y
\end{array}\right],} \\
K=\left[\begin{array}{cccc}
1.824 & 1.704 & 1.346 \\
-1.599 & 2.630 & -0.697 \\
-2.383 & -0.488 & 2.477
\end{array}\right] .
\end{gathered}
$$

Multiple Linear Regression attempts to model the relationship between two or more independent variables and a dependent variable by fitting a linear equation to the observed data [29]. In this implementation, every value of an independent variable (each value of the output voltage of the 


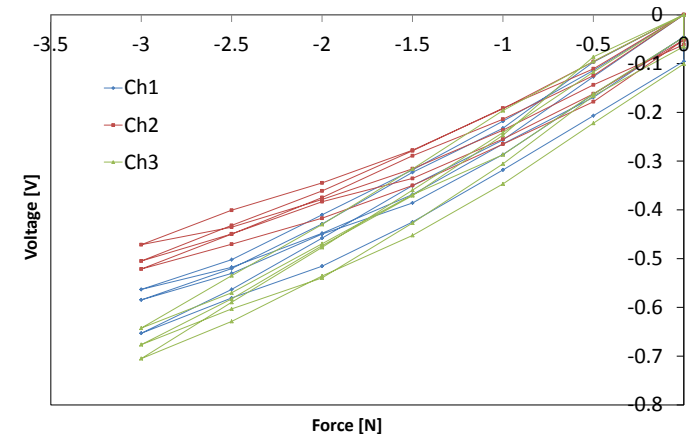

Figure 16. Characteristic curve between the loads $F_{z}$, and the output voltage of the force sensor.

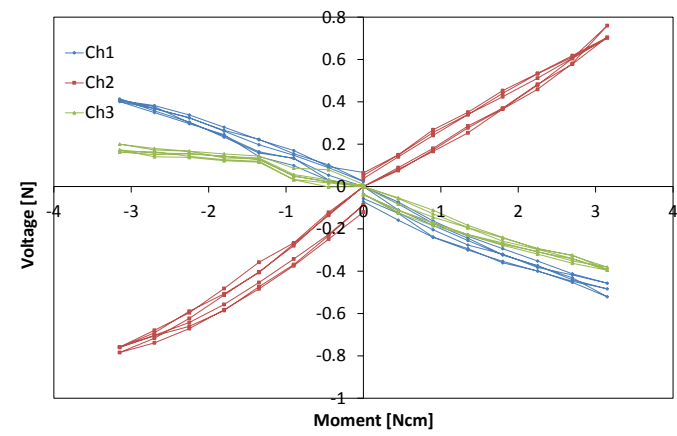

Figure 17. Characteristic curve between the loads $\left(F_{z} M_{x}\right)$ and the output voltage of the force sensor (weights were added to and removed from points (4), (5)) of the calibration device in increments of $(-0.1 \mathrm{~N}$,

$\pm 0.45 \mathrm{Ncm})$ in the range of $(0 \mathrm{~N}, 0 \mathrm{Ncm})$ to $(-0.7 \mathrm{~N}, \pm 3.15 \mathrm{Ncm})$ as shown in Fig. 14 and Table 2)).

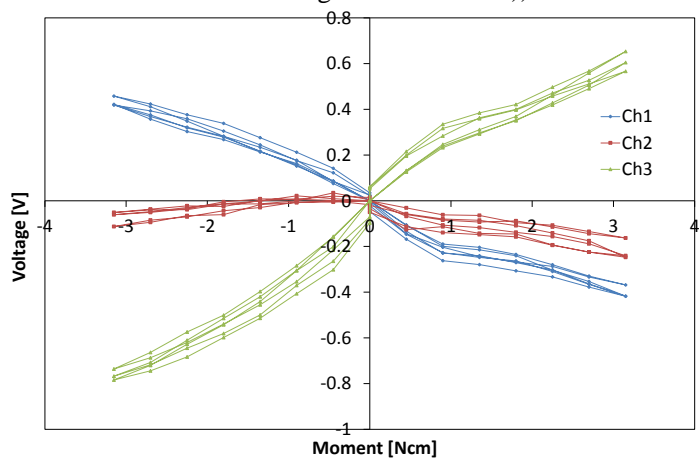

Figure 18. Characteristic curve between the loads $\left(F_{z} M_{y}\right)$ and the output voltage of the force sensor (weights were added to and removed from points (2), (3)) of the calibration device in increments of $(-0.1 \mathrm{~N}$,

$\pm 0.45 \mathrm{Ncm})$ in the range of $(0 \mathrm{~N}, 0 \mathrm{Ncm})$ to $(-0.7 \mathrm{~N}, \pm 3.15 \mathrm{Ncm})$ as shown in see Fig. 14 and Table 2)).

three pairs of the optical fibers) is associated with a value of the dependent variables, force $F_{z}$ and moments $M_{x}$, and $M_{y}$.

By applying Multiple Linear Regression with independent variables $v_{1}, v_{2}$, and $v_{3}$ as shown in Figures 16 to 18 , the stiffness matrix has been calculated for $F_{z}, M_{x}$ and $M_{y}$. As shown in Equations 14 and 15, from the sensor voltage samples such as $v_{1}, v_{2}$, and $v_{3}$, the estimated $F_{z}, M_{x}$, and $M_{y}$ can be obtained through the calculated decoupling stiffness matrix.

\section{Verification of stiffness matrix and objective evaluation} of our proposed three-axis force sensor

In order to objectively evaluate our proposed three-axis force sensor, sensor properties such as crosstalk, hysteresis, repeatability, and error should be examined. For this purpose, a set of experiments were carried out using the calibration device shown in Figures 14 and 15. In order to verify our stiffness matrix and evaluate the sensor properties, the loading/unloading calibration process, (explained in section 3 A. 2) was performed whilst acquiring voltage samples from the three pairs of the optical fibers. Subsequently, the estimated values of $F_{z}, M_{\mathrm{x}}$, and $M_{\mathrm{y}}$ were calculated by multiplying values of voltage samples and stiffness matrix, presented in Eq. 16.

The estimated $F_{z}, M_{\mathrm{x}}$, and $M_{\mathrm{y}}$ were compared with the benchmarks, shown in Figures 19 to 23 and Table 3. It was observed that $M_{\mathrm{y}}$ had slightly higher error terms in comparison with the $F_{z}$ and $M_{\mathrm{x}}$ (Figures 22, 23 and Table 3).

The results of the repeatability test are presented in Table 4, where the ability of the force sensor to reproduce the same condition of the force and of the two moments is demonstrated. It can be seen that each of the force and moment components has a repeatability error less than $3.7 \%$.

The result of the hysteresis is shown in Table 4: loads are applied onto the sensor from zero to the maximum value and then removed in stages. During one cycle, the sensor's characteristic curves exhibit a fairly large hysteresis, as shown in Figures 19 to 23.

The result of the crosstalk is shown in Table 5. When an external force is applied, e.g. $F_{z}$ (force along the $z$-axis), the two moments $M_{x}$ and $M_{y}$ should ideally remain zero. Similarly, if $\left(F_{z} M_{\mathrm{x}}\right)$ is applied on the sensor, $M_{y}$ should ideally remain zero, and if $\left(F_{z} M_{\mathrm{y}}\right)$ is applied on the sensor, $M_{x}$ should remain zero. However, the results from the crosstalk experiments show that the application of a fully loaded force $F_{z}$ or of a force and a moment $\left(\left(F_{z} M_{\mathrm{x}}\right)\right.$ and $\left(F_{z}\right.$ $\left.M_{\mathrm{y}}\right)$ ) along one axis influences the other force/moment readouts, as shown in Figures 19 to 23 and Table 5.

The frequency response is shown in Table 4. Based on the data sheet of the KEYENCETM FS-N11MN, the frequency response of this sensor depends on the chosen light intensity mode and ranges from $62.5 \mathrm{~Hz}$ to $1 \mathrm{kHz}$. This range of frequency response is suitable for the real-time control of the flexible manipulators.

To evaluate the sensor's temperature dependency, the temperature was swept from $30^{\circ} \mathrm{C}$ to $50^{\circ} \mathrm{C}$, measured using a digital thermometer over 23 minutes. The temperature data was synchronized with voltage information provided by the KEYENCETM Fiber Optic Sensor. Table 6 shows the output voltage measured at three temperatures in the range. Although the output voltage of the pair of optical fibers slowly drifts as the temperature changes, the average change of the output voltage of the Fiber Optic Sensor is around $0.03 \mathrm{~V}$, causing the force/moment components' errors to be around $\Delta F_{z}: 0.146 \mathrm{~N}(4.87 \%), \Delta M_{x}: 0.010 \mathrm{Ncm}(0.32 \%)$, and $\Delta M_{y}: 0.011 \mathrm{Ncm}(0.35 \%)$.

\section{CONCLUSION AND FUTURE WORK}

In this paper, we have presented an optics based three-axis force sensor which is capable of measuring externally applied forces and moments and which is particularly suited for integration with a flexible manipulator physically interacting with the environment. We have demonstrated that the design 


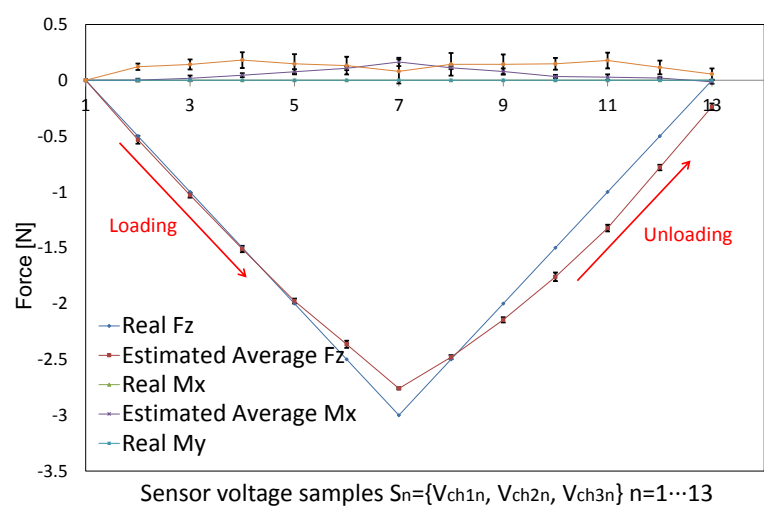

Figure 19. Comparison between real $F_{z}$ values and estimation $F_{z}$

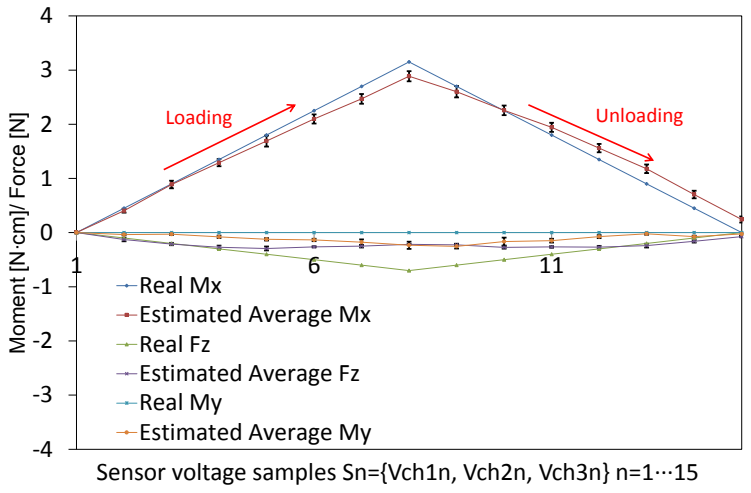

Figure 20. Comparison between real $\left(F_{z} M_{x}\right)$ and estimation $\left(F_{z} M_{x}\right)$ (Positive $M_{x}$ )

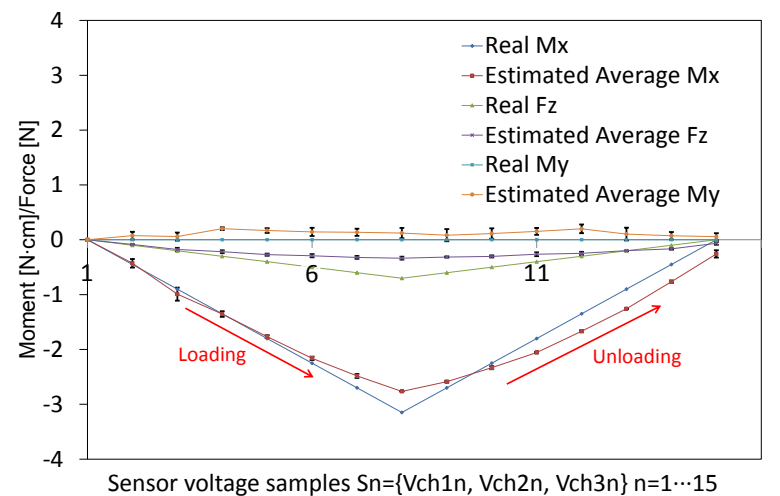

Figure 21. Comparison between real $\left(F_{z} M_{x}\right)$ values and estimation $\left(F_{z} M_{x}\right)$ (Negative $M_{x}$ )

of the sensor structure enables multi axis sensing in a multi-segment flexible manipulator and we have optimized the main parameters of the sensor structure including distance $d$ (between fiber tips and the mirror), angle $a$ (adjustment between fibers), and gap $g$ (between the fibers) in order to obtain an optimized sensing range. In addition, we proposed how to calibrate and calculate a stiffness matrix for a reliable force prediction by applying the multiple linear regression method. Finally, we have validated the effectiveness of our proposed three-axis force sensor through a set of experiments evaluating its properties such as cross-talk, hysteresis, repeatability, error, and temperature dependence.

The errors and the cross-talk are associated with non-linearity, hysteresis, and repeatability. A more close examination reveals that the characteristic curves are not absolutely linear. As shown in Figures 16 and 17, the

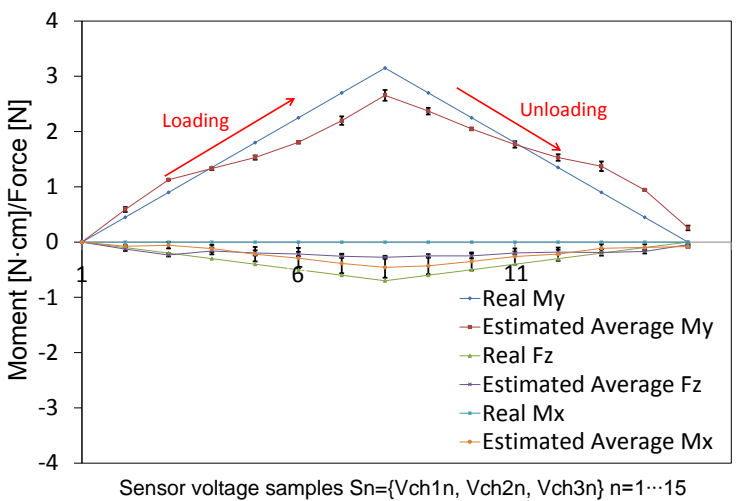

Figure 22. Comparison between real $\left(F_{z} M_{y}\right)$ and estimation $\left(F_{z} M_{y}\right)$ (Positive $M_{y}$ )

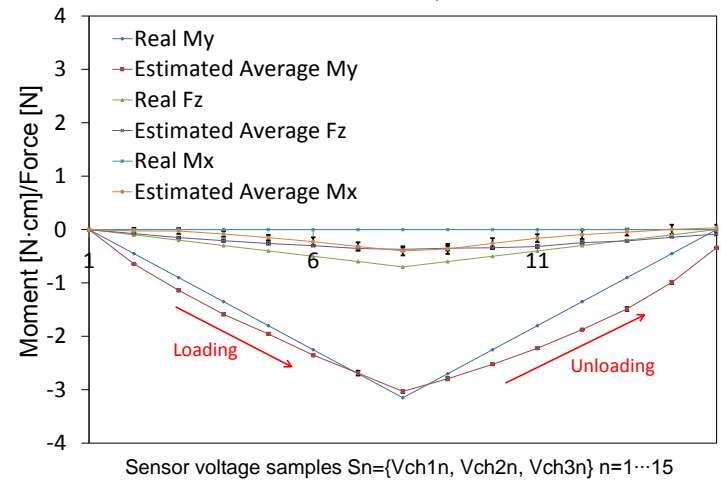

Figure 23. Comparison between real $\left(F_{z} M_{y}\right)$ values and estimation $\left(F_{z} M_{y}\right)$ (Negative $M_{y}$ )

characteristic curves $F_{z}$ and $\left(F_{z} M_{x}\right)$ are fairly linear. The characteristic curve $\left(F_{z} M_{y}\right)$ is not as linear as desired (Figure 18), which results in the maximum errors of the $F_{z}$ and $M_{x}$ being less than $M_{y}$, as shown in Table 3. In addition, the use of Multiple Linear Regression cannot cope with the

TABLE III. SENSOR PERFORMANCE PROPERTY: RANGE AND ERROR

\begin{tabular}{|c|c|c|}
\hline $\begin{array}{c}\text { Force / } \\
\text { Moment }\end{array}$ & Range & Maximum error \\
\hline$F_{z}$ & $+/-3.0 \mathrm{~N}$ & $0.32 \mathrm{~N}(10.7 \%)$ \\
\hline$M_{x}$ & $+/-3.15 \mathrm{Ncm}$ & $0.38 \mathrm{Ncm}(12.2 \%)$ \\
\hline$M_{y}$ & $+/-3.15 \mathrm{Ncm}$ & $0.59 \mathrm{Ncm}(18.2 \%)$ \\
\hline
\end{tabular}

TABLE IV. SENSOR PERFORMANCE REPEATABILITY HySTERESIS AND FREQUENCY RESPONCY

\begin{tabular}{|c|c|c|c|}
\hline $\begin{array}{c}\text { Force / } \\
\text { Moment }\end{array}$ & $\begin{array}{c}\text { Repeatability } \\
\text { Error }\end{array}$ & Hysteresis & $\begin{array}{c}\text { Frequency } \\
\text { response }\end{array}$ \\
\hline$F_{z}$ & $1.2 \%$ & $9.8 \%$ & \multirow{2}{*}{$62.5 \mathrm{~Hz}$ to } \\
\cline { 1 - 2 }$M_{x}$ & $3.7 \%$ & $10.6 \%$ & $1 \mathrm{kHz}$ \\
\hline$M_{y}$ & $3.0 \%$ & $11.2 \%$ & \multirow{2}{*}{} \\
\hline
\end{tabular}

TABLE V. SENSOR PERFORMANCE PROPERTY: CROSS-TALK

\begin{tabular}{|c|c|c|c|}
\hline $\begin{array}{c}\text { Force } / \\
\text { Moment }\end{array}$ & $\boldsymbol{F}_{z}(\boldsymbol{\%})$ & $\left(\boldsymbol{F}_{z} \boldsymbol{M}_{x}\right)(\boldsymbol{\%})$ & $\left(\boldsymbol{F}_{z} \boldsymbol{M}_{\boldsymbol{y}}\right) \mathbf{( \% )}$ \\
\hline$F_{z}$ & $F_{z}$ & $F_{z}$ & $F_{z}$ \\
\hline$M_{x}$ & $5.9 \%$ & $M_{x}$ & $17.2 \%$ \\
\hline$M_{y}$ & $2.8 \%$ & $8.0 \%$ & $M_{y}$ \\
\hline
\end{tabular}

TABLE VI. SENSOR PERFoRMANCE PROPERTY TEMPERATURE

\begin{tabular}{|c|c|c|c|}
\hline $\begin{array}{c}\text { Temperature } \\
\left({ }^{\circ} \mathrm{C}\right)\end{array}$ & $\mathbf{3 0}^{\circ} \mathbf{C}$ & $\mathbf{4 0}^{\circ} \mathbf{C}$ & $\mathbf{5 0}^{\circ} \mathbf{C}$ \\
\hline $\begin{array}{c}\text { Keyence sensor } \\
\text { analog output } \\
(\mathrm{V})\end{array}$ & $2.43 \mathrm{~V}$ & $2.44 \mathrm{~V}$ & $2.46 \mathrm{~V}$ \\
\hline
\end{tabular}


non-linearity properties mentioned above; this influences the calibration matrix thus causing the error of the $F_{z}, M_{x}$, and $M_{y}$ to be higher, as summarized in Table 3. In future work, we will explore the incorporation of nonlinear regression methods to reduce the errors.

Furthermore, due to the hysteresis, the output voltage readings of the three fiber pairs obtained from the loading and unloading processes are not exactly the same (Figures 19 to 23 and Table 4). However, the stiffness matrix is calculated by using the entire set of voltage readings from both loading and unloading, causing the errors in values of the $F_{z}, M_{x}$, and $M_{y}$ to be higher. In addition, as shown in Figures 14 and 15, the sensor calibration base combined with the click-on mechanism could have slight rotation around the $\mathrm{z}$-axis during the loading/unloading of weights, causing inconsistency with the output voltage readings from the three pairs of the optical fibers shown in Figures 16 to 18 . As a result, this small rotation introduces further errors in the calibration matrix calculation, and it negatively influences the repeatability of the sensor.

It should be mentioned that the sensor structure is made of ABS plastic by PROJET VisiJet ${ }^{\circledR}$ EX200, 3D SYSTEM Co., Ltd., a material which causes the hysteresis to be higher in comparison with that experienced in metal structures or in commercial sensors, such as ATI. In the fabrication of future sensors we plan to use MRI compatible metals to counter the issue of high hysteresis.

The temperature influences the sensor's error as shown above. In a constant temperature environment, temperature compensation would not be required. However, since the temperature is changeable in the surgical environment depending on medical devices, temperature compensation should be considered in future designs to measure precise force/torque components.

The sensor presented in this paper is a first prototype intended to demonstrate the mechanism of force/torque measurement. In [25-27], the maximum force when using surgical robots in MIS is shown to range from $0 \mathrm{~N}$ to $21 \mathrm{~N}$, the value depending on the surgical task. Although our proposed force sensor cannot measure the maximum force $21 \mathrm{~N}$, we will be able to build sensor structures which can measure the required force/torque range by choosing design variables $L, E$ (if we use MRI compatible metals, not ABS plastic), $I, b$, and $h$ as shown in Eqs. 4 to 6 and Figure 5,

Regarding the sensor size, the largest size of the trocar's diameter is $15 \mathrm{~mm}$, and so the maximum allowable sensor size should be less than $15 \mathrm{~mm}$. The diameter of our force/torque sensor is around $30 \mathrm{~mm}$, and so the sensor cannot be inserted into the trocars currently. In future designs, our proposed force/torque sensor could be miniaturized, again by choosing design variables $L, E$ (if we use MRI compatible metals, not ABS plastic), $I, b$, and $h$ as shown in Eqs. 4 to 6 and Figure 5 .

As mentioned above, the errors and the cross-talk are associated with non-linearity, hysteresis, and repeatability. The relationship among these parameters will be explored further to improve the sensor design in future work.

\section{REFERENCES}

[1] Amir Degani, Howie Choset, Alon Wolf, Takeyoshi Ota, and Marco A. Zenati, "Percutaneous Intrapericardial Interventions Using a Highly Articulated Robotic Probe", The 2006 IEEE/RAS-EMBS International Conference on Biomedical Robotics and Biomechatronics, pp. 7-12, 2006

[2] Takeyoshi Ota, Amir Degani, David Schwartzman, Brett Zubiate, Jeremy McGarvey, Howie Choset, Marco A. Zenati, "A Highly Articulated Robotic Surgical System for Minimally Invasive Surgery", The Annals of Thoracic Surgery 87(4), 2009, pp. 1253-1256.

[3] N. Simaan, K. Xu, A. Kapoor, W. Wei, P. Kazanzides, P. Flint, and R. Taylor, "A System for Minimally Invasive Surgery in the Throat and Upper Airways", Int. J. Robotics Research (special issue on medical robotics), vol. 28- 9, pp. 1134-1153, June 2009.

[4] Alexander T. Hillel, Ankur Kapoor, Nabil Simaan, Russell H. Taylor and Paul Flint, " Applications of Robotics for Laryngeal Surgery," Laryngeal Cancer -Otolaryngologic Clinics of North America, Nasir Bhatti \& Ralph P. Tufano Eds., Volume 41, Issue 4, Pages 781-791, August 2008.

[5] D. C. Rucker, B. A. Jones, and R. J. Webster III. A Geometrically Exact Model for Externally Loaded Concentrrmfjsic Tube Continuum Robots. IEEE Transactions on Robotics, 26(5), 769-780, 2010.

[6] D. C. Rucker, R. J. Webster III, G. S. Chirikjian, and N. J. Cowan. Equilibrium Conformations of Concentric-Tube Continuum Robots. International Journal of Robotics Research, 29(10), 1263-1280, 2010 (Pre-typesetting preprint) Final definitive version available from IJRR

[7] Zhang, J., Bhattacharyya, S., Simaan, N., "Model and Parameter Identification of Friction During Robotic Insertion of Cochlear-Implant Electrode Arrays," IEEE International Conference on Robotics and Automation (ICRA'2009), pp. 3859-3864, 2009.

[8] Zhang, J., Wei, W., Roland, J., Manolidis, S., Simaan, N., "Optimal Path Planning for Robotic Insertion of Steerable Electrode Arrays in Cochlear Implant Surgery", in ASME Journal on Medical Devices, Vol 3., No. 1, pp. 011001, 2009

[9] Shang, J., Noonan, D.P., Payne, C., Clark, J., Sodergren, M.H., Darzi, A., Yang, G.Z.," An articulated universal joint based flexible access robot for minimally invasive surgery", The 2011 IEEE International Conference on Robotics and Automation (ICRA), pp. 1147-1152, 2011

[10] Noonan, D.P., Vitiello, Valentina, Jianzhong Shang, Payne, C.J., Yang, Guang-Zhong," A modular, mechatronic joint design for a flexible access platform for MIS", 2011 IEEE/RSJ International Conference on Intelligent Robots and Systems (IROS), pp. 949-954, 2011

[11] Noonan, D.P., Mylonas, G.P., Jianzhong Shang, Payne, C.J., Darzi, A., Guang-Zhong Yang, "Gaze contingent control for an articulated mechatronic laparoscope", 2010 3rd IEEE RAS and EMBS International Conference on Biomedical Robotics and Biomechatronics (BioRob), pp. 759-764, 2010.

[12] Arjo J. Loeve, Dick H. Plettenburg, Paul Breedveld, and Jenny Dankelman, "Endoscope Shaft-Rigidity Control Mechanism: "FORGUIDE"”, IEEE Transactions on Biomedical Engineering, 59(2), pp. 542-551, 2012.

[13] N.G. Cheng, M.B. Lobovsky, S.J. Keating, A.M. Setapen, "Design and Analysis of a Robust, Low-cost, Highly Articulated manipulator enabled by jamming of granular media”, The 2012 IEEE International Conference on Robotics and Automation (ICRA), pp. 4328-4333, 2012

[14] P Polygerinos, $\mathrm{P}$ Puangmali, $\mathrm{T}$ Schaeffter, $\mathrm{R}$ Razavi, LD Seneviratne,"Novel miniature MRI-compatible fiber-optic force sensor for cardiac catheterization procedures", The 2010 IEEE International Conference on Robotics and Automation (ICRA 2010), pp. 2598 2603, 2010

[15] P Puangmali, $\mathrm{H}$ Liu, LD Seneviratne, $\mathrm{P}$ Dasgupta, K Althoefer,"Miniature 3-axis distal force sensor for minimally invasive surgical palpation", Mechatronics, IEEE/ASME Transactions on 17 (4), 646-656

[16] Panagiotis Polygerinos, Dinusha Zbyszewski, Tobias Schaeffter, RezaRazavi, Lakmal D Seneviratne, Kaspar Althoefer," MRI-compatible fiber-optic force sensors for catheterization procedures", IEEE Sensors Journal, pp. 1598-1608, 2010

[17] Hui Xie, Hongbin Liu, Yohan Noh, Jianmin Li, Shuxin Wang, Kaspar Althoefer, Fiber-Optics based Body Contact Sensor for a Flexible Manipulator, IEEE Sensors Journal, accepted 2015 
[18] Hongbin Liu, P. Puangmali, D. Zbyszewski, O. Elhage, P. Dasgupta, J. S. Dai, L. Seneviratne, K. Althoefer, "An indentation depth-force sensing wheeled probe for abnormality identification during minimally invasive surgery," Journal of Engineering in Medicine, Proc. Inst. Mech. Eng., H, vol. 224, no. 6, pp. 751-63, 2010.

[19] Min-Gyu Kim, Dong-Hyeok Lee and Nahm-Gyoo Cho, "A force sensor with five degrees of freedom using optical intensity modulation for usage in a magnetic resonance field", Measurement Science and Technology 24, 045101.

[20] R. Monfaredi, R. Seifabadi, G. Fichtinger, I. Iordachita, "Design of a decoupled MRI-compatible force sensor using fiber Bragg grating sensors for robot-assisted prostate interventions", Proc. SPIE 8671, Medical Imaging 2013, 867118.

[21] Matteo Cianchetti, Tommaso Ranzani, Giada Gerboni, Iris De Falco, Cecilia Laschi, Arianna Menciassi," STIFF-FLOP Surgical Manipulator: mechanical design and experimental characterization of the single module", IEEE/RSJ 2013 International Conference on Intelligent Robots and Systems, Nov, 2013

[22] Thomas C. Searle, Kaspar Althoefer, Lakmal Seneviratne, Hongbin Liu," An Optical Curvature Sensor for Flexible Manipulators", The 2012 IEEE International Conference on Robotics and Automation (ICRA), pp. $4401-4405,2012$

[23] Y. Noh, S. Sareh, Jungwhan Back, Helge A Wurdemann, Tommaso Ranzani, Emanuele Lindo Secco, Angela Faragasso, Hongbin Liu, Kaspar Althoefer," A three-axial body force sensor for flexible manipulators", 2014 IEEE International Conference on Robotics and Automation (ICRA), pp. 6388-6393, 2014.

[24] S Sareh, A Jiang, A Faragasso, Y Noh, T Nanayakkara, P Dasgupta, LD Seneviratne, HA Wurdemann, K Althoefer,"Bio-inspired tactile sensor sleeve for surgical soft manipulators", 2014 IEEE International Conference on Robotics and Automation (ICRA), pp. 1454-1459

[25] C. E. Reiley, T. Akinbiyi, D. Burschka, D. C. Chang, A. M. Okamura,and D. D. Yuh, "Effects of visual force feedback on robot-assisted surgical task performance," The Journal of thoracic and cardiovascular surgery, vol. 135, no. 1, pp. 196-202, 2008.

[26] C. R. Wagner, N. Stylopoulos, P. G. Jackson, and R. D. Howe, "The benefit of force feedback in surgery: Examination of blunt dissection," Presence: teleoperators and virtual environments, vol. 16, no. 3, pp. 252-262, 2007.

[27] M. Kitagawa, D. Dokko, A. M. Okamura, and D. D. Yuh, "Effect of sensory substitution on suture-manipulation forces for robotic surgical systems," The Journal of thoracic and cardiovascular surgery, vol. 129, no. 1, pp. 151-158, 2005.

[28] Ferdinand Beer, Jr., E. Russell Johnston, Mechanics of Materials, Paperback (McGraw-Hill), $4^{\text {th }}$ edition.

[29] Laura L. Nathans, Frederick L. Oswald, Kim Nimon, "Interpreting Multiple Linear Regression: A Guidebook of Variable Importance", Practical Assessment, Research \& Evaluation, Vol. 17, No. 9.

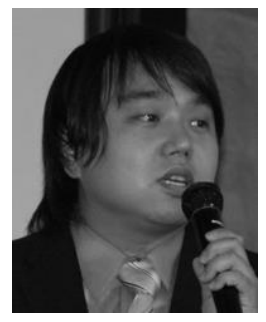

Yohan Noh is a research associate in the Center for Robotics Research (CoRe). He received B.S. degree in the Department of Mechanical Engineering from Seoul National University of Science and Technology, Korea in 2002, B.S. degree in the Department of Electrical Engineering from Yonsei University, Korea in 2004, and received M.S. and Ph.D. degrees in the Department of Science and Engineering from Waseda University, Tokyo, Japan in 2007 and 2011 respectively. His research interests include development of force and tactile sensors, robot assisted ultrasound diagnostic system, and medical robots.

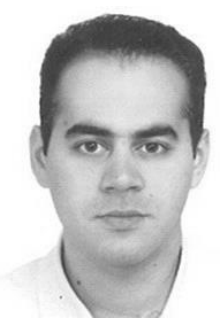

Sina Sareh received the B.S. degree in Electrical Engineering from Amirkabir University of Technology (Tehran Polytechnic), Iran and the M.S. degree in Control Systems from the University of Sheffield, UK, in 2005 and 2007, respectively. He holds a Ph.D. in Robotics from the University of Bristol, UK, 2012. He is currently a research associate in surgical robotics at King's College London. His research focus is on the creation of soft-smart sensors and actuators

for rehabilitation systems and robot-assisted surgery.

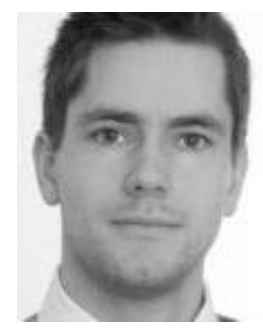

Helge Würdemann received the B.S. degree in Electrical Engineering, and the M.S. degree from Leibniz University of Hanover Amirkabir University in Germany in 2006 and 2008, respectively. He received Ph.D. in Robotics from King's College London in 2012. He is currently a research associate in surgical robotics at King's College London. His interests include the design and application of bio-inspired, soft and stiffness-controllable medical and haptic devices and robotic art. He has published over 20 peer-reviewed papers in journals and at international premium conferences in robotics.

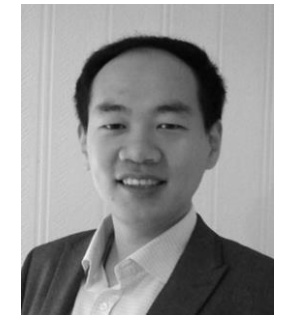

Hongbin Liu is currently a lecturer (Assistant Professor) in the department of Informatics, King's College London, UK. He received his B.S. degree in 2005 from the Northwestern Polytechnique University, Xi'an, China, and was awarded with an MSc degree from King's College London in 2006. He received his $\mathrm{PhD}$ in 2010 from Kings College London. He is a member of IEEE. His research interests include tactile/force perception based robotic cognition, modeling of dynamic interaction, medical robotics and haptics.

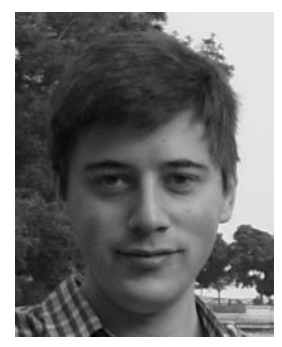

James Housden studied Engineering at the University of Cambridge, UK, and obtained his $\mathrm{PhD}$ in ultrasound imaging from the same university in 2008. From 2008 to 2011 he was with the Department of Engineering at the University of Cambridge, where he worked on ultrasound elasticity imaging. He is currently a research fellow in the Department of Biomedical Engineering, King's College London, where he has worked on robotic and image guidance systems for minimally-invasive surgery. His research interests include ultrasound imaging, image processing and surgical guidance systems.

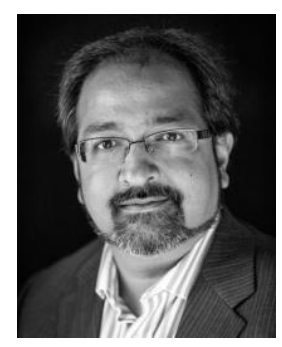

Kawal Rhode obtained his bachelor's degree in Basic Medical Sciences and Radiological Sciences at King's College London in 1992. From 1998 to 2001, he studied for a doctorate at the Department of Surgery, University College London. From 2001 to 2007, Dr. Rhode was employed at the Division of Imaging Sciences, King's College London as a post-doctoral research fellow working in the field of image-guided interventions. In 2007, Dr. Rhode was appointed to Lecturer in Image Processing at King's College London and subsequently to Senior Lecturer in 2011. Dr. Rhode's research interests include image-guided cardiovascular interventions, cardiac electromechanical modelling, computer simulation of minimally invasive procedures and medical robotics.

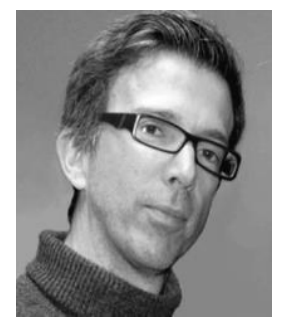

Kaspar Althoefer (M'03) received his Dipl.-Ing. degree in electronic engineering from the University of Aachen, Aachen, Germany, and his $\mathrm{Ph} . \mathrm{D}$. degree in electronic engineering from King's College London, London, U.K. He is currently a Professor of Robotics and Intelligent Systems and Head of the Centre for Robotics Research (CoRe), Department of Informatics, King's College London. He has been involved in research on mechatronics since 1992 and gained considerable expertise in the areas of sensing, sensor signal analysis, embedded intelligence, and sensor data interpretation using neural networks and fuzzy logic, as well as robot-based applications He has authored or coauthored more than 180 refereed research papers related to mechatronics and robotics. 